\title{
Complete genome sequence of the acetate-degrading sulfate reducer Desulfobacca acetoxidans type strain (ASRB2 ${ }^{\mathrm{T}}$ )
}

\author{
Markus Göker ${ }^{1}$, Hazuki Teshima ${ }^{2,3}$, Alla Lapidus ${ }^{2}$, Matt Nolan², Susan Lucas ${ }^{2}$, Nancy \\ Hammon ${ }^{2}$, Shweta Deshpande ${ }^{2}$, Jan-Fang Cheng ${ }^{2}$, Roxanne Tapia ${ }^{2,3}$, Cliff Han ${ }^{2,3}$, Lynne \\ Goodwin $^{2,3}$, Sam Pitluck ${ }^{2}$, Marcel Huntemann ${ }^{2}$, Konstantinos Liolios ${ }^{2}$, Natalia Ivanova ${ }^{2}$, \\ Ioanna Pagani ${ }^{2}$, Konstantinos Mavromatis ${ }^{2}$, Galina Ovchinikova ${ }^{2}$, Amrita Pati ${ }^{2}$, Amy Chen ${ }^{4}$, \\ Krishna Palaniappan ${ }^{4}$, Miriam Land ${ }^{2,5}$, Loren Hauser ${ }^{2,5}$, Evelyne-Marie Brambilla ${ }^{1}$, Manfred \\ Rohde $^{6}$, Stefan Spring ${ }^{1}$, John C. Detter ${ }^{2,3}$, Tanja Woyke ${ }^{2}$, James Bristow ${ }^{2}$, Jonathan A. \\ Eisen $^{2,7}$, Victor Markowitz ${ }^{4}$, Philip Hugenholtz ${ }^{2,8}$, Nikos C. Kyrpides ${ }^{2}$, and Hans-Peter Klenk ${ }^{1 *}$ \\ ${ }^{1}$ DSMZ - German Collection of Microorganisms and Cell Cultures GmbH, Braunschweig, \\ Germany \\ ${ }^{2}$ DOE Joint Genome Institute, Walnut Creek, California, USA \\ ${ }^{3}$ Los Alamos National Laboratory, Bioscience Division, Los Alamos, New Mexico, USA \\ ${ }^{4}$ Biological Data Management and Technology Center, Lawrence Berkeley National \\ Laboratory, Berkeley, California, USA \\ ${ }^{5}$ Oak Ridge National Laboratory, Oak Ridge, Tennessee, USA \\ ${ }^{6} \mathrm{HZI}$ - Helmholtz Centre for Infection Research, Braunschweig, Germany \\ ${ }^{7}$ University of California Davis Genome Center, Davis, California, USA \\ ${ }^{8}$ Australian Centre for Ecogenomics, School of Chemistry and Molecular Biosciences, The \\ University of Queensland, Brisbane, Australia \\ *Corresponding author: Hans-Peter Klenk
}

Keywords: anaerobic, mesophile, organoheterotroph, non-motile, sulfate-reducing, sludge bed reactor, Syntrophaceae, GEBA

Desulfobacca acetoxidans Elferink et al. 1999 is the type species of the genus Desulfobacca, which belongs to the family Syntrophaceae in the class Deltaproteobacteria. The species was first observed in a study on the competition of sulfate-reducers and acetoclastic methanogens for acetate in sludge. D. acetoxidans is considered to be the most abundant acetate-degrading sulfate reducer in sludge. It is of interest due to its isolated phylogenetic location in the $16 \mathrm{~S}$ rRNA-based tree of life. This is the second completed genome sequence of a member of the family Syntrophaceae to be published and only the third genome sequence from a member of the order Syntrophobacterales. The 3,282,536 bp long genome with its 2,969 protein-coding and 54 RNA genes is a part of the Genomic Encyclopedia of Bacteria and Archaea project.

\section{Introduction}

Strain ASRB2 $^{\mathrm{T}}$ (= DSM $11109=$ ATCC 700848) is the type strain of the species Desulfobacca acetoxidans, which is the type and sole species of its genus Desulfobacca [1]. The type strain was isolated from granular sludge of a laboratory-scale upflow anaerobic sludge bed (UASB) reactor fed with acetate and sulfate [1]. The generic name derives from the Neo-Latin word 'desulfo', meaning desulfuricating, and the Latin word ' $b a c c a$ ', berry, especially olive, meaning a sulfate-reducing oliveshaped bacterium. The species epithet is derived from the Neo-Latin words 'acetum', vinegar, and 'oxido', meaning acetate-oxidizing. The strain is important for the understanding of the competition for acetate between sulfate-reducers and acetoclastic methanogens in sludge [1]. Here we present a summary classification and a set of features for D. acetoxidans strain ASRB2T, together with the description of the complete genomic sequencing and annotation.

\section{Classification and features}

The single genomic $16 \mathrm{~S}$ rRNA sequence of $D$. acetoxidans DSM ASRB2 ${ }^{\mathrm{T}}$ was compared using NCBI BLAST [2,3] under default settings (e.g., consider- 
ing only the high-scoring segment pairs (HSPs) from the best 250 hits) with the most recent release of the Greengenes database [4] and the relative frequencies of taxa and keywords (reduced to their stem [5]) were determined, weighted by BLAST scores. The most frequently occurring genera were Desulfobacca (74.9\%) and Desulfomonile (25.1\%) (4 hits in total). Regarding the two hits to sequences from members of the species, the average identity within HSPs was $98.9 \%$, whereas the average coverage by HSPs was $96.7 \%$. Among all other species, the one yielding the highest score was Desulfomonile limimaris (NR_025079), which corresponded to an identity of $90.4 \%$ and an HSP coverage of $49.8 \%$. (Note that the Greengenes database uses the INSDC (= EMBL/NCBI/DDBJ) annotation, which is not an authoritative source for nomenclature or classification.) The highest-scoring environmental sequence was AY340836 ('sulfatereducing fluidized-bed reactor clone SR FBR L13'), which showed an identity of $99.8 \%$ and an HSP coverage of $93.0 \%$. The most frequently occurring keywords within the labels of environmental samples which yielded hits were 'sediment' (5.2\%), 'microbi' (3.2\%), 'lake' (1.9\%), 'water' (1.7\%) and 'depth' (1.6\%) (246 hits in total). The most frequently occurring keywords within the labels of environmental samples which yielded hits of a higher score than the highest scoring species were 'sediment' (5.4\%), 'microbi' (2.5\%), 'lake' (2.1\%), 'water' (1.9\%) and 'contamin' (1.8\%) (152 hits in total). These keywords reflect some of the ecological and properties reported for strain ASRB2 $^{\mathrm{T}}$ in the original description [1].

Figure 1 shows the phylogenetic neighborhood of D. acetoxidans in a $16 \mathrm{~S}$ rRNA based tree. The sequence of the single $16 \mathrm{~S}$ rRNA gene in the genome differs by 20 nucleotides from the previously published 16S rRNA sequence (AF002671), which contains eleven ambiguous base calls.

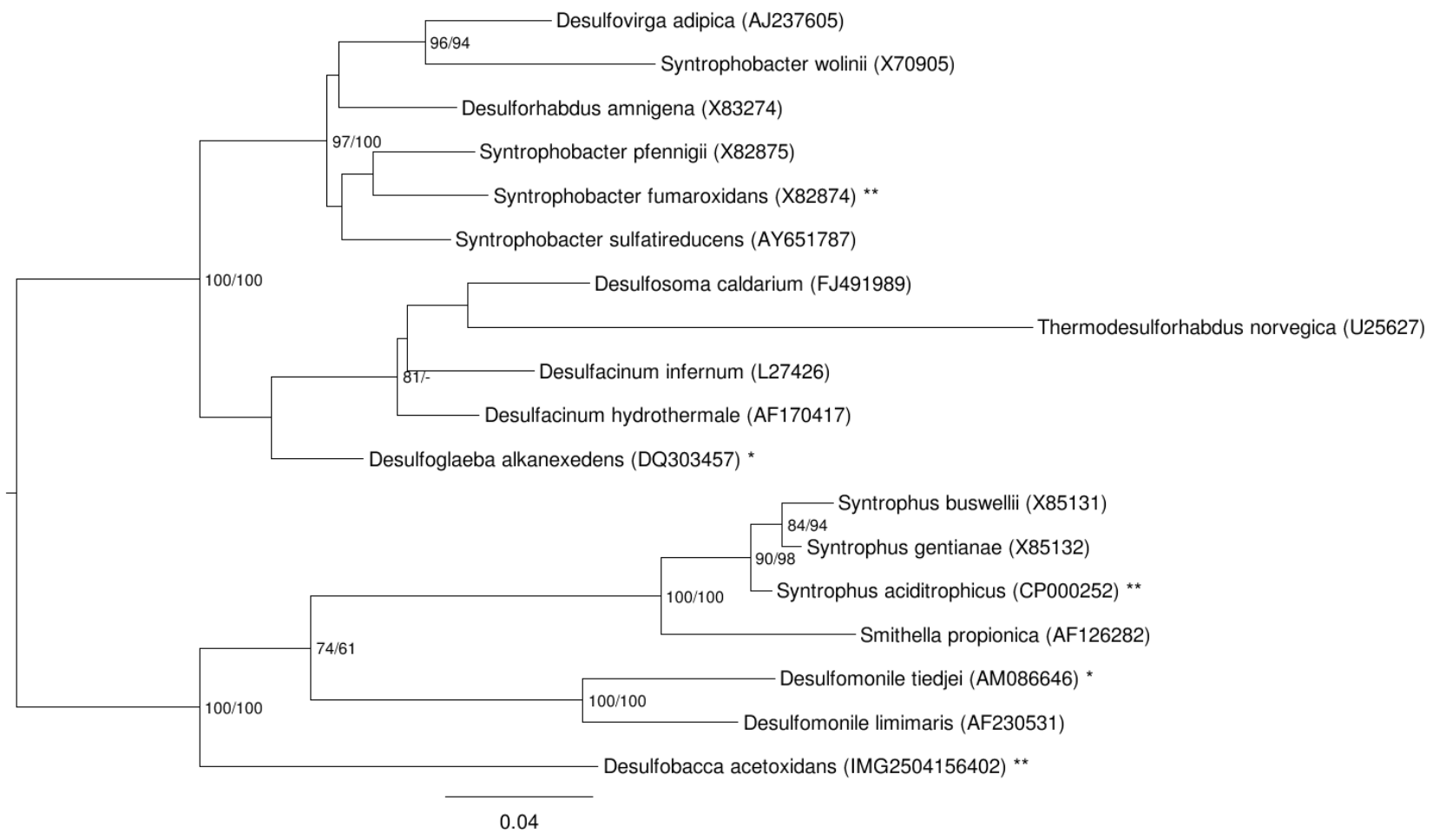

Figure 1. Phylogenetic tree highlighting the position of $D$. acetoxidans relative to the type strains of the other species within the order Syntrophobacterales. The tree was inferred from 1,457 aligned characters [6,7] of the $16 \mathrm{~S}$ rRNA gene sequence under the maximum likelihood (ML) criterion [8]. Rooting was done initially using the midpoint method [9] and then checked for its agreement with the current classification (Table 1). The branches are scaled in terms of the expected number of substitutions per site. Numbers adjacent to the branches are support values from 1,000 ML bootstrap replicates [10] (left) and from 1,000 maximum parsimony bootstrap replicates [11] (right) if larger than 60\%. Lineages with type strain genome sequencing projects registered in GOLD [12] are labeled with one asterisk, those also listed as 'Complete and Published' with two asterisks (see [13] and CP000478 for Syntrophobacter fumaroxidans). 
Goker et al.

Cells of strain ASRB2 ${ }^{\mathrm{T}}$ are oval to rod-shaped with a size of $1.3 \times 1.9-2.2 \mu \mathrm{m}$, appear singly or in pairs (Figure 2) and occasionally contain gas vacuoles in the late-exponential growth phase [1]. The strain is non-motile, non-spore-forming and stains Gramnegative (Table 1) [1]. Strain ASRB2 ${ }^{\mathrm{T}}$ has a temperature range for growth between 27 and $47^{\circ} \mathrm{C}$, with an optimum at $36-40^{\circ} \mathrm{C}$ [1]. At the optimum growth temperature with acetate as sole carbon and energy source the shortest doubling time recorded was 1.7-2.2 days [1]. Growth rate in brackish medium was significantly ( $4.8 \mathrm{x}$ ) slower, and no growth was observed in marine medium [1]. The $\mathrm{pH}$ range for growth is 6.5-8.3, with an optimum of $\mathrm{pH}$ 7.1-7.5 [1]. Desulfoviridin was not observed, but the c-type cytochromes were present [1]. Sulfate or other inorganic sulfur components serve as electron acceptors via reduction to $\mathrm{H}_{2} \mathrm{~S}$ [1]. Strain ASRB2T degrades acetate (as the common carbon source and electron donor) completely to $\mathrm{CO}_{2}$ via the acetylCoA/CO-dehydrogenase pathway [1]. The key enzyme of this pathway is encoded by the genes Desac_1965 - Desac_1969. Several more putative electron donors were tested but not found to be utilized by strain ASRB2 ${ }^{\mathrm{T}}$, such as: propionate, butyrate, lactate, $\mathrm{H}_{2} / \mathrm{CO}_{2}$, formate, ethanol, propanol, butanol, pyruvate, fumarate, glucose, crotonate, benzoate, phenol, aspartate and glutamate [1].

\section{Chemotaxonomy}

No data on cell wall structure, quinones, fatty acid pattern or polar lipids are available for this strain.

\section{Genome sequencing and annotation Genome project history}

This organism was selected for sequencing on the basis of its phylogenetic position [26], and is part of the Genomic Encyclopedia of Bacteria and Archaea project [27]. The genome project is deposited in the Genome On Line Database [12] and the complete genome sequence is deposited in GenBank. Sequencing, finishing and annotation were performed by the DOE Joint Genome Institute (JGI). A summary of the project information is shown in Table 2.

\section{Growth conditions and DNA isolation}

D. acetoxidans ASRB2T, DSM 11109, was grown anaerobically in DSMZ medium 728 (Desulfobacca medium) [28] at $37^{\circ} \mathrm{C}$. DNA was isolated from 0.5$1 \mathrm{~g}$ of cell paste using Jetflex Genomic DNA Purification Kit (GENOMED 600100) following the standard protocol as recommended by the manufacturer, but with additional 2 hours incubation with $20 \mu \mathrm{l}$ proteinase $\mathrm{K}$ at $58^{\circ} \mathrm{C}$ for cell lysis. DNA is available through the DNA Bank Network [29].

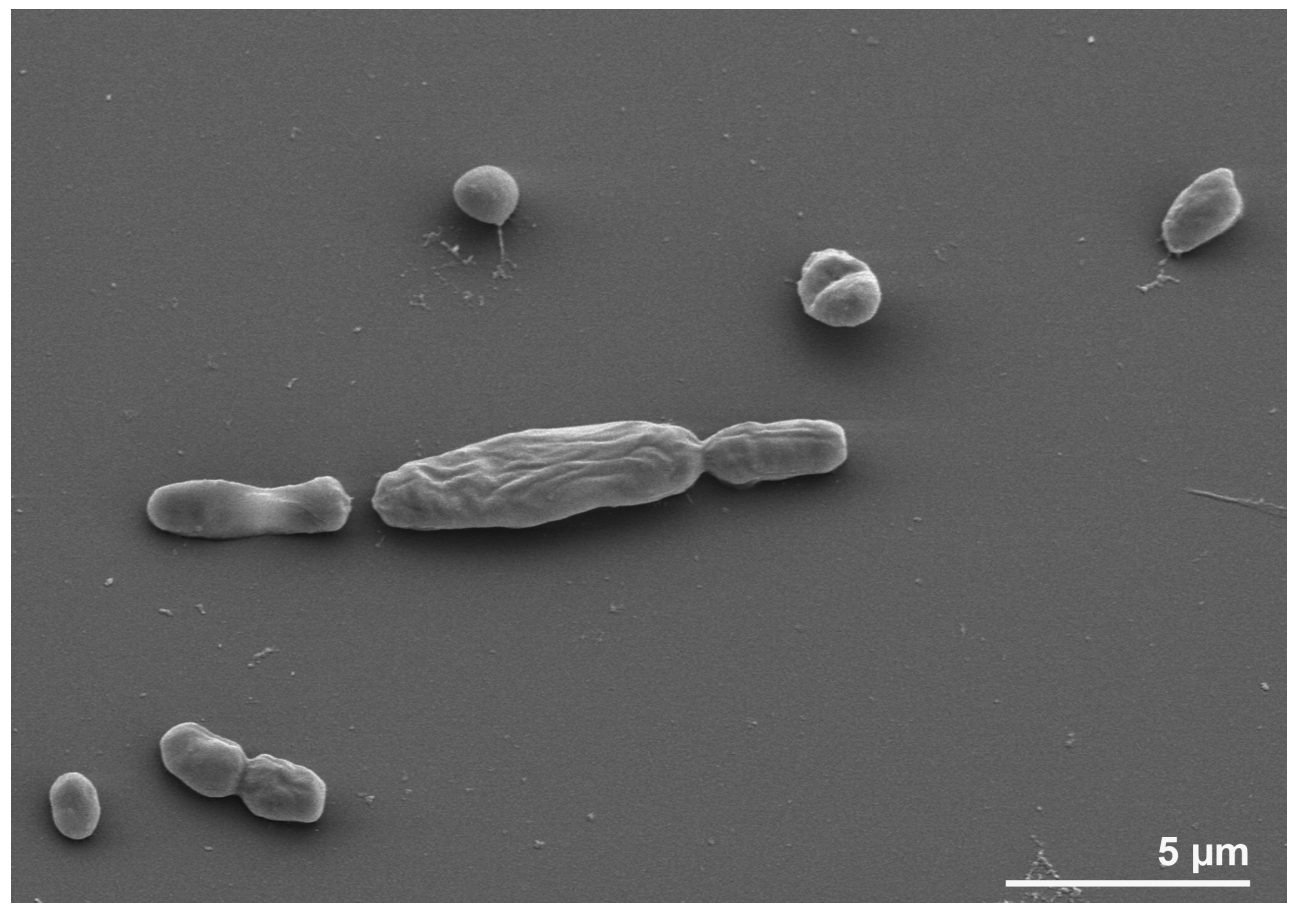

Figure 2. Scanning electron micrograph of D. acetocidans ASRB2 ${ }^{\top}$ 
Table 1. Classification and general features of $D$. acetocidans ASRB2 ${ }^{\top}$ according to the MIGS recommendations [14] and the NamesforLife database [15].

\begin{tabular}{|c|c|c|c|}
\hline MIGS ID & Property & Term & Evidence code \\
\hline & \multirow{8}{*}{ Current classification } & Domain Bacteria & TAS [16] \\
\hline & & Phylum Proteobacteria & TAS [17-19] \\
\hline & & Class Deltaproteobacteria & TAS $[20,21]$ \\
\hline & & Order Syntrophobacterales & TAS $[21,22]$ \\
\hline & & Family Syntrophaceae & TAS $[21,23]$ \\
\hline & & Genus Desulfobacca & TAS [1] \\
\hline & & Species Desulfobacca acetoxidans & TAS [1] \\
\hline & & Type strain ASRB2 & TAS [1] \\
\hline & Gram stain & negative & TAS [1] \\
\hline & Cell shape & oval to rod-shaped & TAS [1] \\
\hline & Motility & none & TAS [1] \\
\hline & Sporulation & none & TAS [1] \\
\hline & Temperature range & $27-47^{\circ} \mathrm{C}$ & TAS [1] \\
\hline & Optimum temperature & $36-40^{\circ} \mathrm{C}$ & TAS [1] \\
\hline & Salinity & low salt conditions & TAS [1] \\
\hline \multirow[t]{3}{*}{ MIGS-22 } & Oxygen requirement & anaerobic & TAS [1] \\
\hline & Carbon source & acetate & TAS [1] \\
\hline & Energy metabolism & organoheterotroph & NAS \\
\hline MIGS-6 & Habitat & fresh water, anaerobic sludge & TAS [1] \\
\hline MIGS-15 & Biotic relationship & free-living & NAS \\
\hline \multirow[t]{3}{*}{ MIGS-14 } & Pathogenicity & none & NAS \\
\hline & Biosafety level & 1 & TAS [24] \\
\hline & Isolation & $\begin{array}{l}\text { anaerobic granular sludge of a pilot-scale UASB reactor } \\
\text { fed with acetate and an excess of sulfate }\end{array}$ & TAS [1] \\
\hline MIGS-4 & Geographic location & Wageningen, The Netherlands & TAS [1] \\
\hline MIGS-5 & Sample collection time & 1995 ore before & NAS \\
\hline MIGS-4.1 & Latitude & 51.97 & TAS [1] \\
\hline MIGS-4.2 & Longitude & 5.67 & TAS [1] \\
\hline MIGS-4.3 & Depth & irrelevant & \\
\hline MIGS-4.4 & Altitude & $25 \mathrm{~m}$ & NAS \\
\hline
\end{tabular}

Evidence codes - IDA: Inferred from Direct Assay (first time in publication); TAS: Traceable Author Statement (i.e., a direct report exists in the literature); NAS: Non-traceable Author Statement (i.e., not directly observed for the living, isolated sample, but based on a generally accepted property for the species, or anecdotal evidence). These evidence codes are from of the Gene Ontology project [25]. If the evidence code is IDA, the property was directly observed by one of the authors or an expert mentioned in the acknowledgements

\section{Genome sequencing and assembly}

The genome was sequenced using a combination of Illumina and 454 sequencing platforms. All general aspects of library construction and sequencing can be found at the JGI website [30]. Pyrosequencing reads were assembled using the Newbler assembler (Roche). The initial Newbler assembly consisting of 66 contigs in one scaffold was converted into a phrap [31] assembly by making fake reads from the consensus, to collect the read pairs in the 454 paired end library. Illumina GAii sequencing data $(1,042 \mathrm{Mb})$ was assembled with Velvet [32] and the consensus sequences were shredded into $1.5 \mathrm{~kb}$ overlapped fake reads and assembled together with the 454 data. The 
454 draft assembly was based on $159.0 \mathrm{Mb} 454$ draft data and all of the 454 paired end data. Newbler parameters are -consed -a 50 -1 350 -g -m -ml 20. The Phred/Phrap/Consed software package [31] was used for sequence assembly and quality assessment in the subsequent finishing process. After the shotgun stage, reads were assembled with parallel phrap (High Performance Software, LLC). Possible mis-assemblies were corrected with gapResolution [30], Dupfinisher [33], or sequencing cloned bridging PCR fragments with subcloning. Gaps between contigs were closed by editing in Consed, by PCR and by Bubble PCR primer walks (J.-F. Chang, unpublished). A total of 55 additional reactions were necessary to close gaps and to raise the quality of the finished sequence. Illumina reads were also used to correct potential base errors and increase consensus quality using a software Polisher developed at JGI [34]. The error rate of the completed genome sequence is less than 1 in 100,000 . Together, the combination of the Illumina and 454 sequencing platforms provided $350.7 \times$ coverage of the genome. The final assembly contained 346,781 pyrosequence and 28,710,424 Illumina reads.

Table 2. Genome sequencing project information

\begin{tabular}{|c|c|c|}
\hline MIGS ID & Property & Term \\
\hline MIGS-31 & Finishing quality & Finished \\
\hline MIGS-28 & Libraries used & 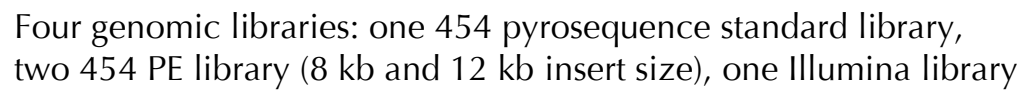 \\
\hline MIGS-29 & Sequencing platforms & Illumina GAii, 454 GS FLX Titanium \\
\hline MIGS-31.2 & Sequencing coverage & $313.2 \times$ Illumina; $37.5 \times$ pyrosequence \\
\hline MIGS-30 & Assemblers & Newbler version 2.3, Velvet 0.7.63, phrap SPS - 4.24 \\
\hline \multirow[t]{6}{*}{ MIGS-32 } & Gene calling method & Prodigal 1.4, GenePRIMP \\
\hline & INSDC ID & СР002629 \\
\hline & Genbank Date of Release & April 15, 2011 \\
\hline & GOLD ID & Gc01720 \\
\hline & NCBI project ID & 51777 \\
\hline & Database: IMG-GEBA & 2504136006 \\
\hline \multirow[t]{2}{*}{ MIGS-13 } & Source material identifier & DSM 11109 \\
\hline & Project relevance & Tree of Life, GEBA \\
\hline
\end{tabular}

\section{Genome annotation}

Genes were identified using Prodigal [35] as part of the Oak Ridge National Laboratory genome annotation pipeline, followed by a round of manual curation using the JGI GenePRIMP pipeline [36]. The predicted CDSs were translated and used to search the National Center for Biotechnology Information (NCBI) non-redundant database, UniProt, TIGRFam, Pfam, PRIAM, KEGG, COG, and InterPro databases. Additional gene prediction analysis and functional annotation was performed within the Integrated Microbial Genomes - Expert Review (IMG-ER) platform [37].

\section{Genome properties}

The genome consists of a 3,282,536 bp long chromosome with a $52.9 \% \mathrm{G}+\mathrm{C}$ content (Table 3 and Figure 3). Of the 3,023 genes predicted, 2,969 were protein-coding genes, and 54 RNAs; 103 pseudogenes were also identified. The majority of the protein-coding genes (68.2\%) were assigned a putative function while the remaining ones were annotated as hypothetical proteins. The distribution of genes into COGs functional categories is presented in Table 4. 


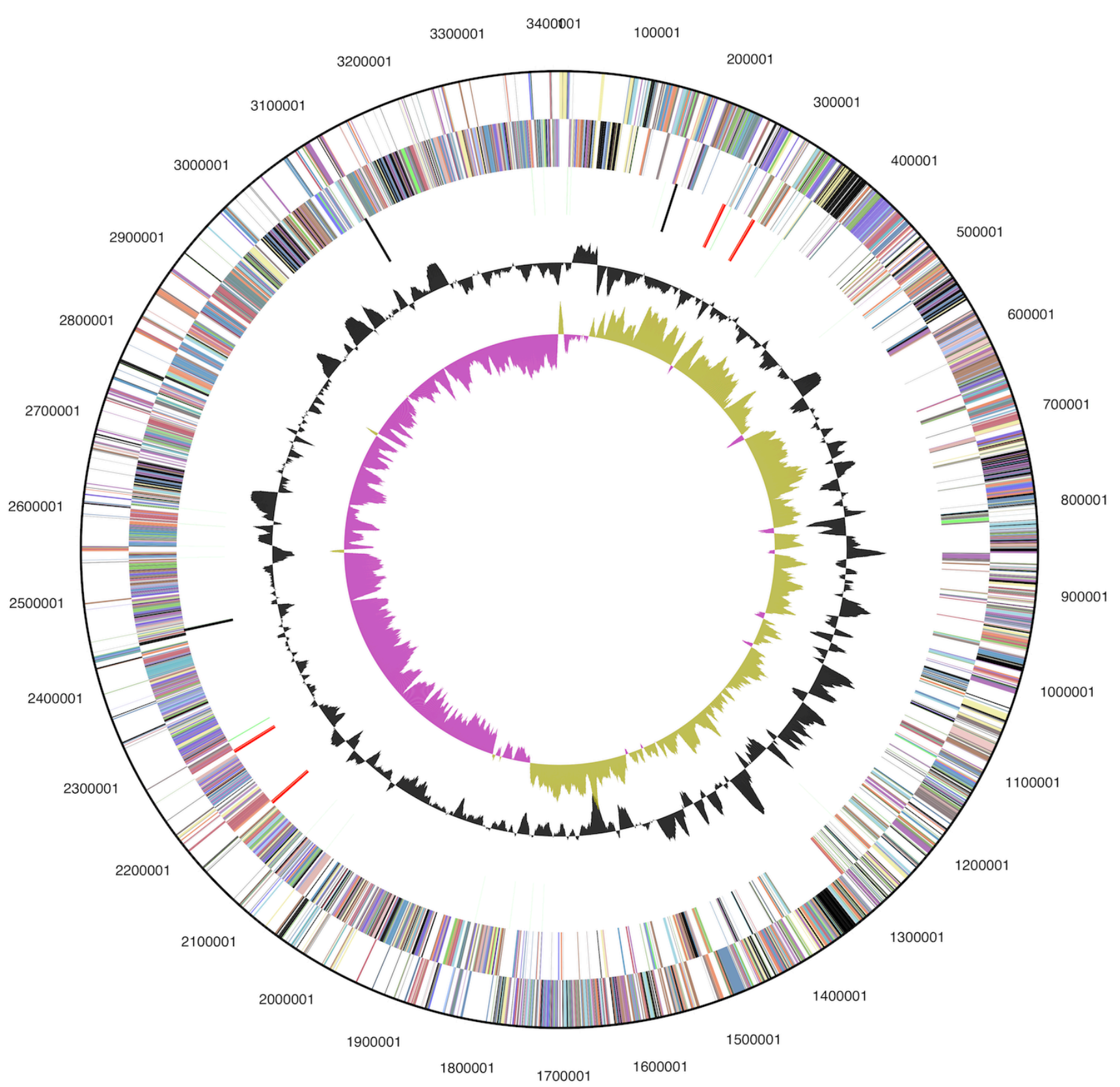

Figure 3. Graphical circular map of the genome. From outside to the center: Genes on forward strand (color by COG categories), Genes on reverse strand (color by COG categories), RNA genes (tRNAs green, rRNAs red, other RNAs black), GC content, GC skew. 
Table 3. Genome Statistics

\begin{tabular}{lrr}
\hline Attribute & Value & \% of Total \\
\hline Genome size (bp) & $3,282,536$ & $100.00 \%$ \\
DNA coding region (bp) & $2,775,726$ & $84.56 \%$ \\
DNA G+C content (bp) & $1,736,170$ & $52.89 \%$ \\
Number of replicons & 1 & \\
Extrachromosomal elements & 0 & \\
Total genes & 3,023 & $100.00 \%$ \\
RNA genes & 54 & $1.79 \%$ \\
rRNA operons & 1 & \\
Protein-coding genes & 2,969 & $98.21 \%$ \\
Pseudo genes & 103 & $3.41 \%$ \\
Genes with function prediction & 2,063 & $68.24 \%$ \\
Genes in paralog clusters & 507 & $16.77 \%$ \\
Genes assigned to COGs & 2,109 & $69.77 \%$ \\
Genes assigned Pfam domains & 2,213 & $73.21 \%$ \\
Genes with signal peptides & 488 & $16.14 \%$ \\
Genes with transmembrane helices & 726 & $24.02 \%$ \\
CRISPR repeats & 4 & \\
\hline
\end{tabular}

Table 4. Number of genes associated with the general COG functional categories

\begin{tabular}{lrrl}
\hline Code & value & \%age & Description \\
\hline J & 158 & 7.0 & Translation, ribosomal structure and biogenesis \\
A & 1 & 0.0 & RNA processing and modification \\
K & 87 & 3.9 & Transcription \\
L & 136 & 6.0 & Replication, recombination and repair \\
B & 3 & 0.1 & Chromatin structure and dynamics \\
D & 27 & 1.2 & Cell cycle control, cell division, chromosome partitioning \\
Y & 0 & 0.0 & Nuclear structure \\
V & 48 & 2.1 & Defense mechanisms \\
T & 140 & 6.2 & Signal transduction mechanisms \\
M & 200 & 8.8 & Cell wall/membrane/envelope biogenesis \\
N & 14 & 0.6 & Cell motility \\
Z & 0 & 0.0 & Cytoskeleton \\
W & 0 & 0.0 & Extracellular structures \\
U & 82 & 3.6 & Intracellular trafficking and secretion, and vesicular transport \\
O & 92 & 4.1 & Posttranslational modification, protein turnover, chaperones \\
C & 189 & 8.4 & Energy production and conversion \\
G & 89 & 3.9 & Carbohydrate transport and metabolism \\
E & 176 & 7.8 & Amino acid transport and metabolism \\
F & 59 & 2.6 & Nucleotide transport and metabolism \\
H & 135 & 6.0 & Coenzyme transport and metabolism \\
I & 49 & 2.2 & Lipid transport and metabolism \\
P & 116 & 5.1 & Inorganic ion transport and metabolism \\
Q & 32 & 1.4 & Secondary metabolites biosynthesis, transport and catabolism \\
R & 262 & 11.6 & General function prediction only \\
S & 167 & 7.4 & Function unknown \\
- & 914 & 30.2 & Not in COGs \\
\hline
\end{tabular}




\section{Acknowledgements}

We would like to gratefully acknowledge the help of Esther Schüler (DSMZ) for growing D. acetoxidans cultures. This work was performed under the auspices of the US Department of Energy Office of Science, Biological and Environmental Research Program, and by the University of California, Lawrence Berkeley National Laboratory under contract No. DE-AC02-05CH11231,

\section{References}

1. Oude Elferink SJ, Akkermans-van Vliet WM, Bogte JJ, Stams AJM. Desulfobacca acetoxidans gen. nov., sp. nov., a novel acetate-degrading sulfate reducer isolated from sulfidogenic granular sludge. Int J Syst Bacteriol 1999; 49:345-350. PubMed doi:10.1099/00207713-49-2-345

2. Altschul SF, Gish W, Miller W, Myers EW, Lipman DJ. Bascic local alignment search tool. J Mol Biol 1990; 215:403-410. PubMed

3. Korf I, Yandell M, Bedell J. BLAST, O'Reilly, Sebastopol, 2003.

4. DeSantis TZ, Hugenholtz $P$, Larsen N, Rojas M, Brodie EL, Keller K, Huber T, Dalevi D, Hu P, Andersen GL. Greengenes, a chimera-checked 16S rRNA gene database and workbench compatible with ARB. Appl Environ Microbiol 2006; 72:5069-5072. PubMed doi:10.1128/AEM.03006-05

5. Porter MF. An algorithm for suffix stripping. Program: electronic library and information systems 1980; 14:130-137.

6. Castresana J. Selection of conserved blocks from multiple alignments for their use in phylogenetic analysis. Mol Biol Evol 2000; 17:540-552. PubMed

7. Lee C, Grasso C, Sharlow MF. Multiple sequence alignment using partial order graphs. Bioinformatics 2002; 18:452-464. PubMed doi:10.1093/bioinformatics/18.3.452

8. Stamatakis A, Hoover P, Rougemont J. A rapid bootstrap algorithm for the RAxML web servers. Syst Biol 2008; 57:758-771. PubMed doi:10.1080/10635150802429642

9. Hess PN, De Moraes Russo CA. An empirical test of the midpoint rooting method. Biol / Linn SoC Lond 2007; 92:669-674. doi:10.1111/j.10958312.2007.00864.x

10. Pattengale ND, Alipour M, Bininda-Emonds ORP, Moret BME, Stamatakis A. How many bootstrap replicates are necessary? Lect Notes Comput Sci
Lawrence Livermore National Laboratory under Contract No. DE-AC52-07NA27344, and Los Alamos National Laboratory under contract No. DE-AC0206NA25396, UT-Battelle, and Oak Ridge National Laboratory under contract DE-AC05-000R22725, as well as German Research Foundation (DFG) INST 599/1-2.

2009; 5541:184-200. doi:10.1007/978-3-642$\underline{02008-7 \quad 13}$

11. Swofford DL. PAUP*: Phylogenetic Analysis Using Parsimony (*and Other Methods), Version 4.0 b10. Sinauer Associates, Sunderland, 2002.

12. Liolios K, Chen IM, Mavromatis K, Tavernarakis $\mathrm{N}$, Hugenholtz P, Markowitz VM, Kyrpides NC. The Genomes On Line Database (GOLD) in 2009: status of genomic and metagenomic projects and their associated metadata. Nucleic Acids Res 2010; 38:D346-D354. PubMed doi:10.1093/nar/gkp848

13. McInerney MJ, Rohlin L, Mouttaki H, Kim U, Krupp RS, Rios-Hernandez L, Sieber J, Struchtemeyer CG, Bhattacharyya A, Campbell JW, Gunsalus RP. The genome of Syntrophus aciditrophicus: Life at the thermodynamic limit of microbial growth. Proc Natl Acad Sci USA 2007; 104:76007605. PubMed doi:10.1073/pnas.0610456104

14. Field D, Garrity G, Gray T, Morrison N, Selengut J, Sterk P, Tatusova T, Thomson N, Allen MJ, Angiuoli SV, et al. The minimum information about a genome sequence (MIGS) specification. Nat Biotechnol 2008; 26:541-547. PubMed doi:10.1038/nbt1360

15. Garrity G. NamesforLife. BrowserTool takes expertise out of the database and puts it right in the browser. Microbiol Today 2010; 37:9.

16. Woese CR, Kandler O, Wheelis ML. Towards a natural system of organisms: proposal for the domains Archaea, Bacteria, and Eucarya. Proc Natl Acad Sci USA 1990; 87:4576-4579. PubMed doi:10.1073/pnas.87.12.4576

17. Garrity GM, Bell JA, Lilburn T. Phylum XIV. Proteobacteria phyl. nov. In: Garrity GM, Brenner DJ, Krieg NR, Staley JT (eds), Bergey's Manual of Systematic Bacteriology, Second Edition, Volume 2, Part B, Springer, New York, 2005, p. 1.

18. Garrity GM, Holt JG. The Road Map to the Manual. In: Garrity GM, Boone DR, Castenholz RW (eds), Bergey's Manual of Systematic Bacteriolo- 
gy, Second Edition, Volume 1, Springer, New York, 2001, p. 119-169.

19. Editor L. Validation of publication of new names and new combinations previously effectively published outside the IJSEM. List no. 106. Int I Syst Evol Microbiol 2005; 55:2235-2238. doi:10.1099/ijs.0.64108-0

20. Kuever J, Rainey FA, Widdel F. Class IV. Deltaproteobacteria class. nov. In: Garrity GM, Brenner DJ, Krieg NR, Staley JT (eds), Bergey's Manual of Systematic Bacteriology, Second Edition, Volume 2, Part C, Springer, New York, 2005, p. 922.

21. Editor L. Validation List No. 107. List of new names and new combinations previously effectively, but not validly, published. Int / Syst Evol Microbiol 2006; 56:1-6. PubMed doi:10.1099/ijs.0.64188-0

22. Kuever J, Rainey FA, Widdel F. Order VI. Syntrophobacterales ord. nov. In: Garrity GM, Brenner DJ, Krieg NR, Staley JT (eds), Bergey's Manual of Systematic Bacteriology, Second Edition, Volume 2, Part C, Springer, New York, 2005, p. 1021.

23. Kuever J, Rainey FA, Widdel F. Family II. Syntrophaceae fam. nov. In: Garrity GM, Brenner DJ, Krieg NR, Staley JT (eds), Bergey's Manual of Systematic Bacteriology, Second Edition, Volume 2, Part C, Springer, New York, 2005, p. 1033.

24. Classification of bacteria and archaea in risk groups. http://www.baua.de TRBA 466.

25. Ashburner M, Ball CA, Blake JA, Botstein D, Butler H, Cherry JM, Davis AP, Dolinski K, Dwight SS, Eppig JT, et al. Gene Ontology: tool for the unification of biology. Nat Genet 2000; 25:25-29. $\underline{\text { PubMed doi:10.1038/75556 }}$

26. Klenk HP, Göker M. En route to a genome-based classification of Archaea and Bacteria? Syst Appl Microbiol 2010; 33:175-182. PubMed doi:10.1016/j.syapm.2010.03.003

27. Wu D, Hugenholtz P, Mavromatis K, Pukall R, Dalin E, Ivanova NN, Kunin V, Goodwin L, Wu $M$, Tindall BJ, et al. A phylogeny-driven genomic encyclopaedia of Bacteria and Archaea. Nature 2009; 462:1056-1060. $\underline{\text { PubMed }}$ doi:10.1038/nature08656
28. List of growth media used at DSMZ: http://www.dsmz.de/microorganisms/media_list.p hp.

29. Gemeinholzer B, Dröge G, Zetzsche H, Haszprunar G, Klenk HP, Güntsch A, Berendsohn WG, Wägele JW. The DNA Bank Network: the start from a German initiative. Biopreservation and Biobanking 2011; 9:51-55. doi:10.1089/bio.2010.0029

30. The DOE Joint Genome Institute. http://www.jgi.doe.gov.

31. Phrap and Phred for Windows. MacOS, Linux, and Unix. http://www.phrap.com.

32. Zerbino DR, Birney E. Velvet: algorithms for de novo short read assembly using de Bruijn graphs. Genome Res 2008; 18:821-829. PubMed doi:10.1101/gr.074492.107

33. Han C, Chain P. 2006. Finishing repeat regions automatically with Dupfinisher. In: Proceeding of the 2006 international conference on bioinformatics \& computational biology. Arabina HR, Valafar H (eds), CSREA Press. June 26-29, 2006: 141-146.

34. Lapidus A, LaButti K, Foster B, Lowry S, Trong S, Goltsman E. POLISHER: An effective tool for using ultra short reads in microbial genome assembly and finishing. AGBT, Marco Island, FL, 2008.

35. Hyatt D, Chen GL, Locascio PF, Land ML, Larimer FW, Hauser LJ. Prodigal: prokaryotic gene recognition and translation initiation site identification. BMC Bioinformatics 2010; 11:119. PubMed doi:10.1186/1471-2105-11-119

36. Pati A, Ivanova NN, Mikhailova N, Ovchinnikova G, Hooper SD, Lykidis A, Kyrpides NC. GenePRIMP: a gene prediction improvement pipeline for prokaryotic genomes. Nat Methods 2010; 7:455-457. PubMed doi:10.1038/nmeth.1457

37. Markowitz VM, Ivanova NN, Chen IMA, Chu K, Kyrpides NC. IMG ER: a system for microbial genome annotation expert review and curation. Bioinformatics 2009; 25:2271-2278. PubMed doi:10.1093/bioinformatics/btp393 